\title{
'Berliner' Ballen und 'Potsdamer' Bürgermeister - Substantive oder Adjektive?
}

Wortformen wie Berliner und Potsdamer treten in pränominaler attributiver Funktion auf: eine Position, in der sowohl Adjektive als auch Substantive stehen können. Substantive kommen in der Position vor als sächsische Genitive (Leos Auto), als vorangestellte Genitivattribute (des Vaters Pflicht) oder als Bestandteile einer engen Apposition (Bundeskanzler Schröder). Adjektive stehen an dieser Stelle als adjektivische Attribute (rotes Auto). Gegen jede dieser Interpretationen von Berliner sprechen jeweils formale Argumente, die im wesentlichen darauf hinauslaufen, daß Berliner in Berliner Ballen niemals flektiert wird - weder wie ein Substantiv noch wie ein Adjektiv.

Welcher Wortart sind Wortformen wie Berliner in Berliner Ballen also zuzuordnen? Zur Beantwortung dieser Frage folgen zunächst einige (kommentierte) Literaturstellen, anschließend werde ich die Bezeichnung 'Stadtadjektive' einführen, ich nehme also zum Zwecke der Benennung eine Entscheidung vorweg. Darauf folgt die Untersuchung: das Verhalten der Stadtadjektive in Bezug auf Flexion, Derivation, Komposition und Syntax. ${ }^{\prime}$

\section{Das Problem in der Literatur}

Sowohl die Interpretation als Adjektiv als auch als Substantiv werden in der Literatur vertreten. Zur Illustration werden drei Zitate angeführt, die aus drei verschiedenen Bänden der Duden-Reihe stammen.

Im Rechtschreib-Duden kommen die Fälle in den Regeln der Groß- und Kleinschreibung vor. Obwohl es in dem Paragraphen um Adjektive geht, werden die entsprechenden Formen nur "Wörter" genannt.

Die von geographischen Namen abgeleiteten Wörter auf -er schreibt man immer groß: das Ulmer Münster, cine Kölner Firma, eine Schweizer Industric, der Holländer Käse.

(Duden 1, 1996: 40)

Gegensätzliche Positionen beziehen der Grammatik-Duden und der ZweifelsfälleDuden. Unter dem Abschnitt "unflektierte attributive Adjektive" wird in dem Grammatik-Duden auch der hier behandelte Fall genannt:

1 Dieser Aufsatz ist als Vorfassung gedacht; das letzte Wort ist hier noch nicht gesagt und auch die Lösung ist als vorläufig zu betrachten. Eine solche Vorfassung entspricht dem intendierten Charakter der Working Papers. Speziell in diesem Fall ist sic aber - im Sinne des Vorworts - eine Vorfassung für Ewald Lang, denn ich hoffe sehr auf seine Kommentare. 
Ursprünglich ein Substantiv (eigentlich ein Genitiv Plural des betreffenden Einwohnernamens als vorangestelltes Genitivattribut) ist auch die von einem Orts- oder Ländernamen abgeleitete Form auf -er, die heute als attributives flexionsloses Adjektiv aufgefasst wird.

(Duden 4, 1998: 260)

Der Zweifelsfälle-Duden hingegen versucht, die Formen als Substantive zu etablieren.

\begin{abstract}
Ableitungen von Ortsnamen auf -er wie Münch(e)ner Oktoberfest (zu München), Berliner (zu Berlin), Frankfurter (zu Frankfurt), Wiener (zu Wien) werden immer großgeschrieben, d.h. auch in Fügungen wie Münch(e)ner Oktoberfest, Berliner Zeitung, Frankfurter Würstchen, Wiener Walzer. Es handelt sich hier um Substantive in der Funktion eines vorangestellten Genitivattributs: Münch(e)ner Oktoberfest - Oktoberfest der Münch(c)ner (=Genitiv Plural der substantivischen Einwohnerbezeichnung). Diese Bildungen unterscheiden sich damit grundsälzlich von scheinbar gleich gebauten Wörtern wie deutscher und österreichischer in Fügungen wie deutscher Michel und österreichischer Beitrag, die Formen der Adjektive deutsch bzw. österreichisch darstellen und deshalb kleinzuschreiben sind. Also: eine deutsche (zu deutsch), eine österreichische (zu österreichisch) und eine Schweizer (zu Schweiz) Botschaftsangestellte.

(Duden 9, 1997: 229)
\end{abstract}

Abgesehen von der 'ratgebenden' Funktion der Duden-Bände, wo eben über Großschreibung und auch Zusammenschreibung (bei Straßennamen) entschieden werden muß, taucht das Problem in der Literatur ansonsten eher am Rande auf. Sugarewa (1974) behandelt zwar ausführlich die Ableitungen auf -er und -isch und stellt auch die Unterschiede der beiden Typen sorgfältig dar, diskutiert aber letztendlich nicht die Möglichkeit, er-Ableitungen in der angegebenen Position als Substantive zu interpretieren. Bei ihr sind es also Adjektive.

Die Adjektive vom Typ italienisch, Berliner, Schillersch bilden eine Subgruppe auf der semantischgrammatischen Ebenc der Wortart Adjektiv.

(Sugarewa 1974: 200)

Die Einordnung als Adjektiv folgt bei ihr aus der Stellung bzw. der Funktion, dabei behandelt sie das Suffix -er sogar als Adjektivsuffix.

Bemerkenswert ist, daß das Suffix $-e r$, mit dessen Hilfe Adjektive von geographischen Namen gebildet wcrden, nicht imstande ist, von sich aus die Wortartzugehörigkeit des Wortsyntagmas zu kennzeichnen. Die Einordnung in die Wortklasse des Substantivs oder des Adjektivs wird unter Berücksichtigung der Relationen im Satz-Syntagma oder Wortgruppen-Syntagma möglich: Die Leizpziger sind stolz auf ihre Geschichte. Die Leipziger Messe ist von großer Bedeutung für die Entwicklung der Stadt.

(Sugarewa 1974: 204f)

Sie erläutert in diesem Zusammenhang Besonderheiten dieses Suffixes im Vergleich mit $-i s c h$.

Der Adjektivierung stehen die Derivationsmorpheme -er und -isch zur Verfügung. Sie verhalten sich morphologisch und syntaktisch verschieden. Die -er-Bildungen sind nur auf attributiven Gebrauch beschränkt. Sie sind unveränderlich. Dadurch nähern sie sich der kleinen Gruppe von nichtflektierbaren Adjektiven wie orange, lila, prima, extra

(Sugarewa 1974: 253)

Fleischer (1982: 256) nennt die Formen zunächst Substantive, zeigt aber, daß nichts "gegen die Behandlung der Formen als Adjektive" spricht. Zwingend wird die Einordnung für Fleischer dadurch, daß manche der Bildungen nur attributiv vorkommen, die homonyme Bewohnerbezeichnung fehlt. 
Diese Substantive können auch in attributiv adjektivischer Funktion auftreten, vgl. die Leipziger Messe, die Berliner Einwohnerschaft, der Rostocker Hafen. Weder die Großschreibung (infolge der Homonymie mit den substantivischen Bewohnerbezeichnungen) noch die Unflektierbarkeit (sie gilt auch für Adjektive wic barfuß, rosa u.ä.) sprechen gegen die Bchandlung der Formen als Adjektive. Sie wird schließlich auch deshalb gefordert, weil es von Eigennamen abgeleitete -er-Derivate gibt, die als Substantive gar nicht gebräuchlich sind, vgl. z.B. Teutoburger Wald (aber nicht: *der, die Teutohurger), Böhmer Wald (substantivische Bewohnerbezeichnung: der Böhme), Jasmunder Bodden (Rügen), [...] Umgckehrt werden nicht alle substantivischen Bewohnerbezeichnungen als Adjektive verwendet: Neben der Gruppe ein Schweizer Geschäftsfreund (mit Adjektiv) steht nicht ein Cherusker Dorf [...], auch die Engländer Sprache ist nicht möglich.

Die Adjeklive auf -er sind vielfach an die Stelle älterer Bildungen auf -isch getreten (Fleischer 1982: 256)

Auch andere Wortbildungen behandeln -er wie Sugarewa als Adjektivsuffix (z.B. Motsch 1999: 216f., Simmler 1998: 561, Altmann/Kemmerling 2000); Motsch diskutiert dies ausführlicher, s. unten.

\section{Adjektiv oder Substantiv?}

Um die zu untersuchenden Formen benennen zu können, nehme ich eine Entscheidung bezüglich der Wortart vorweg, die durch die Andeutung der Argumente naheliegt: es finden sich Argumente für und gegen die Interpretation als Adjektiv und solche gegen die Interpretation als Substantiv, aber letztendlich spricht kein Argument für die Interpretation als Substantiv. Danach ist eine Benennung 'Adjektiv' nahegelegt, wenn auch keineswegs endgültig gerechtfertigt. Im folgenden werden Formen wie Berliner in Konstruktionen wie Berliner Ballen STADTADJEKTIVE genannt. 'Stadt' aus folgendem Grund: Ableitungen von Städten und von Ländern unterscheiden sich offenbar. Für die Bildung von Einwohnerbezeichnungen von Ländern stehen zwei Suffixe und beide jeweils noch mit einem Interfix (Stammallomorphie) zur Verfügung, also vier unterschiedliche 'Endungen': -er, -an-er, - $e$, -es-e (zu weiteren Erläuterungen und zur Verteilung s. Fuhrhop 1998: 141ff.). Die entsprechenden Adjektive werden jeweils mit -isch gebildet: Ind-er - ind-isch, Schwed-e - schwed-isch, Chin-es-e - chin-es-isch, Amerik-an-er-amerik-an-isch.

Die Ableitung von Städtenamen geschieht meistens durch das Suffix -er ohne Stammallomorphie: Berliner, Kölner, Potsdamer, Moskauer ${ }^{2}$, New Yorker, Pariser, Wuppertaler usw. Stammallomorphie findet sich nur in wenigen Ausnahmen: Hallenser, Hannoveraner, Jenenser. Die er-Ableitungen bezeichnen zunächst die Bewohner; diese Bewohnerbezeichnungen sind eindeutig Substantive. Entsprechende Adjektive auf -isch finden sich kaum; berlinisch, kölsch, wienerisch. Sie werden nicht einheitlich gebildet (berlinisch mit dem gesamten Städtenamen, kölsch durch Abspaltung eines Segments des Städtenamens, wienerisch durch Einfügen von -er) und sie bezeichnen bevorzugt die Sprachvariante der Bewohner ${ }^{3}$. So erklärt sich auch die gleichzeitige

2 Moskowiter gilt nach Duden (1996) als veraltet.

3 Auch chinesisch usw. kann für die Sprache stehen, aber eben nicht ausschließlich, chinesische Mauer usw. 
Existenz von Stadtadjektiven auf -er von diesen Städtenamen: Berliner Ballen, Kölner Karneval, Wiener Würstchen.

Ableitungen von Städte- und Länderbezeichnungen unterscheiden sich also wesentlich in zweierlei Hinsicht:

1) Bewohnerbezeichnungen von Städtenamen werden mit -er gebildet ohne Stammallomorphie, für die Bewohnerbezeichnungen von Ländern stehen hingegen vier Endungsvarianten zur Verfügung.

2) Adjektive von Länderbezeichnungen werden mit Hilfe von -isch gebildet, diese Bildungen verhalten sich ganz wie Adjektive. Von Städtebezeichnungen können keine Adjektive auf -isch gebildet werden, die eine allgemeine Zugehörigkeit ausdrücken.

2.1 Flexion des adjektivischen Attributes und der engen Apposition
a) der Berliner Platz, des Berliner Platzes, die Berliner Plätze, den Berliner Plätzen
b) der heitere Junge, des heiteren Jungen, die heiteren Jungen, den heiteren Jungen
c) der Bundeskanzler Schröder, des Bundeskanzler(s) Schröder, die ehemaligen Bundeskanzler Brandt und Schmidt, den ehemaligen Bundeskanzlern Brandt und Schmidt.
d) der Potsdamer Tobias, des Potsdamer(s) Tobias, die Potsdamer Tobias und Robert, den Potsdamern Tobias und Robert

In a) ist die Flexion (bzw. die Nichtflexion) von Berliner in attributiver Stellung gezeigt, der für uns interessante Fall. Zur Gegenüberstellung zeigt b) die 'normale' attributive Adjektivflexion mit einem Adjektiv, das wie die Stadtadjektive auf -er endet. Dieses Beispiel zeigt, daß es keine segmentalphonologischen Gründe für die Nichtflektierbarkeit von Stadtadjektiven gibt.

In c) und d) handelt es sich um enge Appositionen, das heißt um Nominalgruppen mit zwei Substantiven, die beide Kern der Nominalgruppe sein können. In der engen Apposition werden die Substantive 'normal' flektiert. Aufgeführt sind die Fälle aus mindestens zwei hier wesentlichen Gründen. Erstens: Sollte es sich bei den Stadtadjektiven doch um Substantive handeln, so wäre die enge Apposition eine naheliegende Interpretation. Der zweite Grund ist eine Formgleichheit: zu den Stadtadjektiven gibt es häufig die gleichlautende Bewohnerbezeichnung, die eindeutig ein Substantiv ist (die Berliner gehen heute wählen). Und mit diesen Substantiven gibt es eben auch eine von der Wortstellung her gleiche Wortgruppe. Dennoch sind die Fälle deutlich zu unterschieden. Die Konstruktion mit dem Substantiv verhält sich stets völlig systemkonform, das heißt wie andere Substantive auch. Die Stadtadjektive hingegen verhalten sich weder wie Substantive noch wie Adjektive, sondern bilden zunächst einmal eine Sonderklasse.

\subsection{Unflektierte attributive Adjektive}

Die Stadtadjektive in 2.1 a) flektieren also weder wie Substantive noch wie Adjektive. Diese Tatsache ist der eigentliche Ursprung des gesamten Problems. Im heutigen Deutschen finden sich noch zwei weitere Typen von Fällen, in denen attributiv unflektierte Adjektive stehen können. 
Der erste Typ sind einzelne Wendungen, in denen ein Adjektiv unflektiert aussieht wie in wie kein schöner Land zu dieser Zeit; Abendrot, gut Wetter droht; trocken Brot ${ }^{4}$. Derartige Fälle sind historisch durch eine Veränderung im Flexionssystem zu erklären, denn die im heutigen Deutschen unflektierte Kurzform ( $g u t)$ des Adjektivs entsprach im älteren Deutschen der Nominativ-Singular-Form in allen drei Genera (Paul 1917: 163). Für das heutige Deutsche sehen die Adjektive in diesen Fällen unflektiert aus, dieser Typ ist aber nicht produktiv.

Der zweite Typ sind einzelne Adjektive, die nicht flektiert werden, bzw. nicht unbedingt flektiert werden müssen: rosa, lila, orange, türkis, aubergine, pink - klasse, spitze, extra, scheiß, mist. Ein türkis Kleid ist deutlich eher zu akzeptieren als *ein blau Kleid.

Für die Adjektive, die auf einen betonbaren Vokal enden, also extra, lila, rosa wird häufig eine phonologische Begründung für die Nichtflektierbarkeit angenommen: In der regulären Flexion würde eine Hiatus entstehen, der offenbar vermieden wird wie in *ein rosaes Auto. Wird es dennoch flektiert, wie es durchaus vorkommt, wird ein - $n$ eingeschoben: ein rosanes Auto.

Für klasse und spitze nennt Eisenberg (mündliche Mitteilung) eine andere Erklärung, ebenfalls phonologisch-morphologischer Art: Seit dem Mittelhochdeutschen haben die Adjektive auf Schwa systematisch ihr Schwa in der Kurzform abgestoßen, bis auf eine kleine Restklasse. Diese Restklasse ist wohlbestimmt, sie enthält nur solche Adjektive, die vor dem Schwa einen stimmhaften Obstruenten haben (Paul 1917: 165), also dröge, leise, müde, öde, träge, trübe, weise. Sie behalten ihr Schwa gewissermaßen, um die Auslautverhärtung zu vermeiden (Eisenberg, mündliche Mitteilung). Kommt nun ein auf Schwa auslautendes Adjektiv ins System (hier durch Konversion), das als vorletzten Laut keinen stimmhaften Obstruenten aufweist, dann ist das Deutsche offenbar nicht fähig, dieses in die normale Adjektivflexion aufzunehmen (so Eisenberg, mündliche Mitteilung). Für diese Interpretation spricht auch, daß später entlehnte Adjektive auf Schwa auslauten können, wenn vorher ein stimmhafter Obstruent steht, so marode ${ }^{5}$, prüde. Sie werden problemlos in das Flexionssystem integriert. klasse und spitze können zwar attributiv vorkommen, bleiben aber unflektiert. Andere Adjektive, die auf Schwa auslauten ohne einen vorangehenden stimmhaften Obstruenten, können hingegen nicht attributiv verwendet werden wie kirre und pleite; *eine pleite Firma, *ein pleites Unternehmen. Die einzige Ausnahme, die sich im rückläufigen Wörterbuch findet (Theissen et al. 1992) ist irre; es kommt attributiv vor und wird flektiert: ein irres Gefühl. Viele Wörterbücher nennen hier aber auch ein Variante ohne Schwa: irr, damit widerspricht auch dieser Fall nicht der ausgeführten These.

Einige Fälle von Nichtflektierbarkeit können also phonologisch-morphologisch erklärt werden; von den oben genannten Adjektiven werden aubergine, orange, pink, türkis; scheiß und mist davon nicht erfaßt. Sie teilen folgende Gemeinsamkeit: sie sind alle durch Konversion aus Substantiven entstanden (außer pink). Dies gilt auch für die bereits phonologisch erklärten mit der Ausnahme von extra. extra ist ein konvertiertes Adverb.

Die Nichtflektierbarkeit der Stadtadjektive kann wie gesagt nicht segmentalphonologisch begründet werden. Aber auch sie werden durch Konversion von Substantiven

${ }^{4}$ Für dieses Beispiel danke ich Wolfgang Ulirich Wurzel.

5 marode ist im 18. Jahrhundert aus dem Französischen entlehnt worden (Pfeifer), und es hat noch nicht einmal in der Schrift ein $-e$ im Französischen. 
gebildet. Dazu ist zunächst festzuhalten, daß die oben genannte Gruppe von Adjektiven, die durch Konversion aus Substantiven entstanden sind, sehr klein ist und sie durch die Aufnahme der Stadtadjektive um einiges vervielfacht würde. Zwei Fragen stellen sich: Wie häufig kommt eine Konversion von Adjektiv zu Substantiv vor? Sind diese zunächst immer in ihrer Flexion beschränkt oder handelt es sich doch um Ausnahmen?

Grundsätzlich gibt es präferierte Konversionsrichtungen, die typologisch und auch für das Deutsche gelten. Eschenlohr (1999: 46ff.) faßt zusammen, daß Konversionen vom Substantiv zum Verb, vom Verb zum Substantiv und vom Adjektiv zum Verb häufig und produktiv sind. Den Wortartenwechsel von Infinitiven und Partizipien diskutiert sie; hier wäre eine Erweiterung wiederum vom Verb zum Substantiv anzunehmen und eine vom Verb zum Adjektiv. Auch wenn Fuhrhop/Teuber (2000) einen Unterschied zwischen den beiden Partizipien herausstellt, so zeigt doch die Auflistung, daß Konversionen vom Substantiv zum Adjektiv gänzlich unüblich sind. Auch Wilmanns (1930: 509f.) begründet das auffallende Verhalten der Stadtadjektive mit der ungewöhnlichen Konversionsrichtung ,und selten haben es die so entstehenden Wörter zu einer vollen Entfaltung adjectivischer Formen gebracht".

Die Konversion von Substantiven zu Adjektiven ist auch typologisch stark eingeschränkt, es handelt sich dabei stets um Einzelfälle.

Zusammenfassend läßt sich also festhalten, daß sowohl phonologische Begründungen als auch die ungewöhnliche Konversionsrichtung Gründe für die Nichtflektierbarkeit von Adjektiven in attributiver Stellung sein können.

Die zunächst augenfällige Gemeinsamkeit von Adjektiven des Typs klasse, rosa und der Stadtadjektive war die Unflektierbarkeit. Als weitere Gemeinsamkeit kommt nun die Entstehung hinzu. Der Entstehensprozeß ist eine wesentliche Ursache für die Unflektierbarkeit. Daher spricht die Existenz von anderen unflektierbaren Adjektiven für die Interpretation der Stadtadjektive als Adjektive. Der Unflektierbarkeit wird somit als Argument gegen die 'Adjektivität' die wesentliche Argumentationskraft genommen.

\subsection{Form der Adjektivsuffixe}

In Fuhrhop (1998: 89) habe ich gezeigt, warum ein Adjektivsuffix möglichst keine Schwasilbe sein sollte. Dies hat insbesondere mit der Form der Komparations- und Flexionssuffixe zu tun; regulär können dabei zwei Schwasilben aufeinander folgen: größ-er - größ-er-es. Wäre das Derivationssuffix auch eine Schwasilbe, so käme es regulär zu drei aufeinanderfolgenden Schwasilben und damit zu vierhebigen Füßen, die für das Deutsche eher untypisch sind (Eisenberg 1991). Einige Adjektive, die auf Schwasilben enden, hat das Deutsche, wie munter, heiter, edel u.a. Bei diesen kann die erste Schwasilbe synkopiert werden: muntreres; wäre dies das Derivationssuffix, so würde das Derivationssuffix unsilbisch werden, was offenbar verhindert wird. Die hier diskutierten Fälle widersprechen diesem Präferenzgesetz keineswegs, denn erstens ist das -er kein Adjektivsuffix, sondern es handelt sich um konvertierte komplexe Substantive, -er ist ein Substantivsuffix. Damit widerspreche ich explizit Sugarewa (1974), die -er als Adjektivsuffix behandelt (s. 1.). Außerdem flektieren die Wortformen ja gerade nicht. Ein Zusammenhang zwischen dem Nichtflektieren und der Form der 
diskutierten Fälle ist zwar möglich, aber nicht offensichtlich, denn heiter, munter usw. flektieren ja auch. Ein Zusammenhang zwischen der Form der Suffixe und dem Nichtflektieren wäre einfach herzustellen, aber er ist letztendlich nicht zu beweisen, da es keine vergleichbaren Fälle gibt.

Nun habe ich behauptet, daß Adjektivsuffixe keine Schwasilben sind, also keine grundsätzlich unbetonbaren Silben. Aber auch -isch bekommt kaum einen Nebenakzent; für die prosodische Struktur der entstehenden Wortformen sind -er und -isch also gewissermaßen gleich schlecht. In diesem Sinne argumentiert Becker (1824), daß -er in gewissen Sinne dem -isch vorgezogen wird, da die Adjektive auf -er nun mal unflektierbar sind:

Die Namen der Länder und Oerter sind nämlich meistens mil Berg, Burg, Dorf, Stadt, Gau, Land, Feld, Heim, Haus u.s.f. zusammengesetzt wie Nürnberg, Marburg u.s.f. Die von ihnen gebildeten Adjektive auf isch haben, weil auf die erste volltonige Sylbe eine zweite halbtonige und noch eine dritte unbetonte Sylbe folgt, für sich schon keinen guten Rhythmus. Nimmt das Adjektiv nun noch eine unbetonte Biegungsendung an, so entsteht ein Verstoß gegen den Rhythmus, welchen die Sprache nicht wohl erträgt, und der, wenn der Ortsname gar dreisylbig ist, ganz unleidlich wird.

(Becker 1824: 318)

Hier ist nun ein weiterer tendenzieller Unterschied zwischen Ableitungen von Städten und Ländern festzuhalten, der mit Becker die unterschiedliche Adjektivbildung erhellt: Ländernamen haben sehr häufig eine Endung, die abgespalten wird (insbesondere -ien); daher entstehen 'bessere' prosodische Strukturen: Inder - indisch. Außerdem ziehen die beiden Interfixe -an- und -es- (amerikán, chinés) den Akzent auf sich. Die Stammformbildung der Länderbezeichnungen ermöglicht also häufig erst ein Adjektiv auf -isch.

\subsection{Argumente aus der Derivation}

Daß es sich bei Berliner in Berliner Ballen nicht um die Personenbezeichnung handeln kann, ist unter anderem daran zu erkennen, daß es nicht movierbar ist. Es ist auch nicht movierbar in Fällen, in denen es von der Bedeutung her sinnvoll wäre: *Berlinerin Bürgerin. Enge Appositionen, in denen Berliner eindeutig ein Substantiv ist, movieren wie in Bundeskanzlerin Merkel oder die Potsdamerin Stefanie. Die Nichtmovierbarkeit spricht gegen die Interpretation der Stadtadjektive als Substantive.

Von den meisten Länderbezeichnungen wird ein entsprechendes Adjektiv mit -isch gebildet, von Städtebezeichnungen nicht. Einige der Länderbezeichnungen bilden hingegen ähnlich wie Städte ihre Einwohnerbezeichnungen auf - er; diese können aber nicht pränominal attributiv (ohne Artikel) verwendet werden: Potsdamer Bürgermeister *Inder Präsident. Insofern füllt Potsdamer zumindest eine Lücke; es gibt kein anderes konkurrierendes Adjektiv. Auch dies spricht für die Interpretation von Potsdamer als Adjektiv.

Die er-Ableitungen sind im Gegensatz zu den isch-Ableitungen weder komparierbar (*die berlinerer Bevölkerung) noch mit un-präfigierbar (*die unberliner Bevölkerung) (Sugarewa 1974: 204). Auch für die isch-Adjektive von Länderbezeichnungen scheinen die Beispiele konstruiert (sein unamerikanisches Verhalten, sein inzwischen deutlich amerikanischeres Verhalten oder er ist jetzt schon amerikanischer als ich es je 
geworden bin). Stadtadjektive unterliegen als Adjektive formalen Beschränkungen, sie sind gewissermaßen weniger adjektivisch als sie sein könnten.

Sugarewa (1974) führt als Argument noch an, daß er-Adjektive nicht substantivierbar seien, also schön - der/das/die Schöne. Dies ist nun an dieser Stelle nicht überraschend, da - sollten Berliner und Potsdamer Adjektive sein - es sich um konvertierte Adjektive aus Substantiven handelt. Daß diese wiederum zu Substantiven konvertieren, ist nicht zu erwarten.

\subsection{Komposita}

Einige Punkte der vorangehenden Analyse könnten dazu führen, Berliner Bürgermeister usw. als Kompositum zu analysieren. Zunächst stehen hierfür die Regeln zur Rechtschreibung, wie sie im Duden zur Getrennt- und Zusammenschreibung von Straßennamen aufgeführt werden (s.2.5.2). Bekanntermaßen werden Straßennamen wie Berliner Straße getrennt geschrieben; der Regelapparat im Duden braucht dazu eine spezielle Regel, die aus dem sonstigen Regelapparat heraus nicht verstanden werden kann, sie muß auswendig gelernt werden.

In der neueren Literatur finden sich bezüglich der Interpretation als Kompositum gegensätzliche Standpunkte, die aber möglicherweise regional bedingte Unterschiede zeigen (insbesondere in Bezug auf den Akzent).

In der Südwestecke des deutschen Sprachraums, der Schwciz, werden Verbindungen mit Ableitungen von geographischen Eigennamen auf -er [...] häufig nicht als Juxtapositionen, sondern als Komposita aufgefaßt; in geschriebener Sprache äußert sich dies in der Zusammenschreibung. In Straßennamen o.ä. ist diese Schreibung in vielen Städten sogar amtlich:

die Schweizeralpen, die Schweizergrenze

die Schaffhauserstraße, der Altstetterplatz, die Wipkingerbrücke (alle in Zürich) (Gallmann 1990: 312)

Motsch (1999: 216, ähnlich auch Henzen 1957: 164) weist auf die unterschiedliche Akzentstruktur hin:

könnten dic Konstruktionen nur als N+N-Komposita analysiert werden. Von normalen Komposita unterscheiden sich die Konstruktionen jedoch dadurch, dass der Hauptakzent nicht auf dem ersten Nomen, sondern auf dem zweiten liegt. Dies wiederum ist die typische Intonation von Konstruktionen aus attributivem Adjektiv und Bezugsnomen in Nominalphrasen.

Wegen des Akzentverhaltens und der völlig freien (und durchsichtigen) Kombinatorik meine auch ich, daß es sich nicht um Komposita handelt. Möglicherweise tendieren Straßennamen als festere Einheiten in diese Richtung, Konstruktionen wie Berliner Bürgermeister - und um die geht es hier wesentlich - nicht.

Historisch sind die Stadtadjektive wie gesagt aus vorangestellten Genitivattributen entstanden. So sind auch die sogenannten Genitivkomposita entstanden, aus denen dann die Fugenelemente hervorgegangen sind (s. z.B. Demske 1996, Fuhrhop 1998 u.a.): des Gottes Diener - der Gottesdiener. Aus analogen Konstruktionen sind zwei unterschiedliche synchrone Konstruktionen entstanden. Es folgen nun einige Überlegungen zur Interpretation dieses Tatbestandes. 


\subsubsection{Berliner als Kompositumserstglied?}

Für Adjektive als Erstglieder gibt es eine formale Beschränkung: Suffigierte Adjektive sind als Erstglieder nicht zugelassen (*Künstlichhonig, *Farbiggraphik, Reis 1983: 114, Wunderlich 1986). Diese Beschränkung ist bei Adjektiven mit einem Adjektivsuffix zu beobachten. -er ist aber kein Adjektivsuffix, sondern es ist ein Substantivsuffix. Denn auch wenn die Stadtadjektive tatsächlich Adjektive sein sollten, so sind sie doch durch Konversion aus Substantiven entstanden und nicht direkt durch Suffigierung. Ob solche Fälle aufgrund der gleichen Begründung von der Komposition ausgeschlossen sind, wie suffigierte Adjektive, ist letztendlich schwer zu prüfen, denn es ist ein singulärer Fall. Es könnte sein, aber es könnte auch nicht sein.

Aber es ist eben in dieser Beziehung noch eine weitere interessante Beobachtung zu machen. Denn auch als eindeutige Substantive, das heißt als Bewohnerbezeichnungen, sind sie von der Komposition ausgeschlossen: *Berlinerclique. Bewohnerbezeichnungen, die mit Hilfe anderer Suffixe gebildet sind, können Kompositionserstglieder sein, wie Chinesenclique, Polenmarkt, Franzosenbillichkeit (eine Anekdote von Kleist). Der in 2. erwähnte Unterschied zwischen den Adjektiven von Städtenamen und Länderbezeichnungen schlägt hier unter Umständen bis in diese Beschränkung hinein, so scheint ?Italienerclique zumindest besser als *Berlinerclique. Auch ? Berlinerinnenclique scheint akzeptabler.

Offenbar hat die Nichtkomponierbarkeit der Substantive Berliner usw. nichts mit der Form des Suffixes zu tun, denn Lehrerzimmer, Bäckermeister, Anglerzubehör sind voll akzeptierte Komposita. Die Beschränkung muß etwas damit zu tun haben, daß Adjektive und Substantive formgleich sind.

In folgender These könnte eine Begründung stecken (sofern sie denn richtig ist): Komposita unterscheiden sich auch formal von Phrasen, eine unterschiedliche Akzentstruktur reicht im allgemeinen nicht aus.

a) Adjektivische Erstglieder sind unflektiert in Komposita, auch bei flektierbaren Adjektiven: Grünkern - grüner Kern.

b) Verben erscheinen als Kompositionserstglied in der Form des Verbstammes: Backform.

c) Vorangestellte Substantive sind entweder sächsische Genitive von Eigennamen (Leos Auto) oder Genitivattribute mit Artikel (des Mannes liebstes Spielzeug).

Beim vorangestellten Genitivattribut kann es systematisch zu Formgleichheiten kommen, wenn nämlich der Genitiv-Plural ohne spezielle Endung gebildet wird, was genau der Fall ist, wenn der Plural ohne Endung gebildet wird und das passiert unter anderem bei Maskulina auf -er. Hier ist die Stelle, in der es zu Formgleichheiten kommen kann, eben der Berliner Bürgermeister als Bürgermeister der Berliner oder als der Bürgermeister von Berlin. In der hier besprochenen Konstruktion wird der Artikel immer vom Kernsubstantiv bestimmt. Ein Kompositum hätte also stets die gleiche Form wie die Phrase, sofern das Zweitglied maskulin ist und im Singular steht (möglicherweise würden sie sich in der Akzentstruktur unterscheiden).

Komposita wie Haustür, Backform, Grünkern können also nur Komposita und keine Phrasen sein. Da Berliner auch gleichzeitig ein Adjektiv sein kann, als solches nicht flektiert wird und als Attribut stets einem Substantiv vorangehen kann, ist auch die Komposition mit dem (eindeutigen) Substantiv verhindert, wegen der Formgleichheit. Die Homonymie hat also auch Auswirkungen auf das Verhalten des Substantivs. 


\subsubsection{Straßennamen}

Für Straßennamen findet sich folgende Regel im Duden:

Straßennamen werden zusammengeschrieben, wenn sic aus einem ungebeugten Adjektiv und einem Grundwort zusammengesetzt sind

(Duden 1, 1996: 43)

Wären unsere Untersuchungsobjekte unflektierte (ungebeugte) Adjektive, wovon wir ausgehen, dann würde dies also zur Zusammenschreibung führen.

Darunter findet sich die Sonderregel, daß Straßennamen auf -er getrennt geschrieben werden. Dies wird zurecht als Sonderregel dargestellt, denn begründet werden kann es innerhalb der Regeln nicht, wenn es auch suggeriert wird. Denn ist das Erstglied ein Substantiv (worauf verwiesen wird), dann würde dies zur Zusammenschreibung führen.

Die Regel im Duden wäre einfach zu verändern, statt "ungebeugte Adjektive" sollte es "ungebeugte, aber beugbare Adjektive" heißen. Die hier untersuchten Formen sind eben nicht nur unflektiert sondern unflektierbar und das ist ein wesentlicher Unterschied.

\subsection{Syntaktische SteIlung der Stadtadjektive}

\subsubsection{Stadtadjektive im attributiven Gebrauch}

Im Gegensatz zu den isch-Adjektiven sind die er-Ableitungen weder prädikativ noch adverbial zu verwenden: er kocht amerikanisch - *er kocht Berliner.

Er ist amerikanisch, er bleibt amerikanisch - Er ist Berliner, er bleibt Berliner sind grammatisch, aber nur in der Interpretation als Substantiv, dann ist es zum Beispiel auch movierbar, sie ist Berlinerin, sie bleibt Berlinerin und es ist artikelfähig ich bin ein Berliner. Auch dies ist kein Gegenargument zu einer Interpretation als Adjektiv, denn es gibt viele Adjektive, die nur attributiv verwendet werden können: die semantische Theorie, ?die Theorie ist semantisch, ?er spricht semantisch (Eisenberg, mündliche Mitteilung).

\subsubsection{Stellung der Stadtadjektive}

Sie können mit anderen Adjektiven kombiniert werden. Die Stadtadjektive stehen dabei unmarkierterweise direkt vor dem Kernsubstantiv der Nominalphrase: die großen Berliner Zeitungen - ?die Berliner großen Zeitungen. Die IdS-Grammatik (Zifonun et al. 1997: 2070f.) gibt eine relative Ordnung pränominaler attributivischer Adjektive an ${ }^{6}$ : "Herkunftsadjektive stehen in unmarkierter Stellung nach allen anderen". Die Regularität gilt also nicht nur für die Stadtadjektive, sondern gleichermaßen für Herkunftsadjektive auf -isch. Bei anderen Herkunftsadjektiven ist dies jedoch nur die unmarkierte Stellung, ein Verstoß dagegen führt keineswegs zu ungrammatischen Konstruktionen. Bei einer 'Kontrastbetonung' ist die umgekehrte Reihenfolge möglich: die neue

${ }^{6}$ Für diesen Hinweis danke ich George Smith. 
italienische Mode - die italienische neue Mode. Laut Gallmann (1990: 189) "können Ableitungen auf -er von geographischen Eigennamen nicht vom folgenden Nomen getrennt werden". Als Beispiel gibt er lediglich

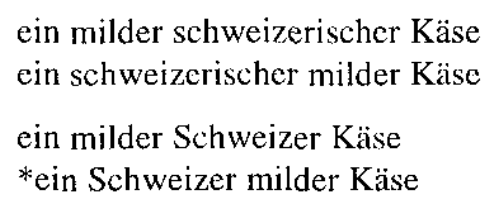

Nun bezeichnet Schweizer Käse eben nicht nur einen Käse aus der Schweiz, sondern häufig eine ganz besondere Sorte von Käse. Insofern nenne ich hier ein anderes Beispiel, das möglicherweise doch ein anderes attributives Adjektiv zwischen Stadtadjektiv und Substantiv zuläßt: 'Die Berliner großen Zeitungen versuchen den Berliner kleinen Zeitungen Leser abzuwerben. Eventuell ist das Stadtadjektiv noch ein wenig unbeweglicher als vergleichbare isch-Adjektive; ein grundsätzlicher Unterschied ist an dieser Stelle nicht eindeutig festzustellen.

Untereinander sind die Stadtadjektive aneinanderreihbar. In den folgenden Beispielen handelt es sich stets um Straßennamen (das zweite Stadtadjektiv in Verbindung mit dem Kernsubstantiv).

Der Berliner Potsdamer Platz ist nahe dem Berliner Pariser Platz und weit entfernt von der Potsdamer Berliner Straße.

Hier handelt es sich um die nähere Bestimmung von benannten Plätzen. Dies führt dazu, $\mathrm{da} ß$ die Reihenfolge der er-Ableitungen wesentlich für die Bedeutung ist. Das liegt wohl eher an der Bedeutung und ist kein wirklich grammatisches Phänomen: ein Platz liegt entweder in Potsdam oder Berlin und wenn ein Potsdamer Platz in Berlin liegt, dann nur deswegen, weil jemand ihn so genannt hat.

\subsubsection{Genitivattribut}

Entstanden ist die Konstruktion aus einem vorangestellten Genitivattribut, das heute durchaus noch grammatisch ist, wenn auch kaum noch gebräuchlich (oder wie Steche 1925: 315 es ausdrückt, "die heute nur noch in der Edelsprache üblich ist"). Daß es sich bei unseren Konstruktionen nicht um ein Genitivattribut handelt (im Gegensatz zu der Angabe im Zweifelsfälle-Duden), ist formal insbesondere am Artikel zu erkennen. Wir haben hier nicht die Konstruktion der Potsdamer Bürgermeisterin betrachtet, sondern die Potsdamer Bürgermeisterin. Der Artikel ist dabei regiert vom Kernsubstantiv und nicht Teil eines vorangestellten Genitivattributs. 


\section{Vorläufiger Schluß}

Die Argumentation zeigt, daß an dieser Stelle wohl mehr Argumente für die Interpretation als Adjektiv sprechen, ohne daß es letztendlich entschieden werden kann.

Die Argumente, die dagegen sprechen, sind die Nichtflektierbarkeit, die Nichtkomparierbarkeit und die Unmöglichkeit der Präfigierung mit un-. Die Nichtflektierbarkeit teilen sie mit einer Reihe von Adjektiven, die ebenfalls durch Konversion aus Substantiven entstanden sind. Die Nichtkomparierbarkeit und Unmöglichkeit der Präfigierung mit un- teilen sie zum Beispiel mit dem 'adjektivischen Infinitiv', dem Partizip 1 (Fuhrhop/ Teuber 2000).

\section{Literatur}

Altmann, Hans; Kemmerling, Silke (2000): Wortbildung fürs Examen. Wiesbaden: Westdeutscher Verlag Becker, Karl Ferdinand (1824): Die deutsche Wortbildung oder die organische Entwickelung der deutschen Sprache in der Ableitungen. Nachdruck der Ausgabe Frankfurt am Main, 1824. Hildesheim: Olms, 1990

Demske, Ulrike (1998): Case compounds in the history of German. In: Butt, Matthias; Fuhrhop, Nanna: Variation und Stabilität in der Wortstruktur. Hildesheim: Olms. (=Germanistische Linguistik 141142). 150-176

Duden 1 (1996): Die Rechtschreibung. 21. Auflage. Mannheim: Duden

Duden 4 (1998): Grammatik. 6. Auflage. Mannheim: Duden

Duden 9 (1997): Zweifelsfalle der deutschen Sprache. 4. Auflage. Mannheim: Duden

Eisenberg, Peter (1991): Syllabische Struktur und Wortakzent. Prinzipien der Prosodik deutscher Wörter. Zeitschrift für Sprachwissenschaft 10. 37-64

Eschenlohr, Stefanie (1999); Vom Nomen zum Verb. Hildesheim: Olms

Fuhrhop, Nanna (1998): Grenzfälle morphologischer Einheiten. Tübingen: Stauffenburg

Fuhrhop, Nanna; Teuber, Oliver (2000): Das Partizip 1 als adjektivischer Infinitiv. In: Biltner, Andreas; Bittner, Dagmar; Köpcke, Klaus-Michael: Angemessene Strukturen: Systemorganisation in Phonologie, Morphologie und Syntax. Festschrift für Wolfgang Ullich Wurzel. Hildesheim: Olms: 173-190

Fleischer, Wolfgang (1982): Wortbildung der deutschen Gegenwartssprache. 5. Auflage. Tübingen: Niemeyer Gallmann, Peter (1990): Kategoriell komplexe Wortformen. Tübingen: Niemeyer

Henzen, Walter (1957): Deutsche Wortbildung. 2. Auflage. Tübingen: Niemeyer

Motsch, Wolfgang (1999): Deutsche Wortbildung in Grundziigen. Berlin; New York: Walter de Gruyter

Paul, Herman (1917): Deutsche Grammatik II. Tübingen: Niemeyer, 1968

Pfeifer, Wolfgang (1993): Etymologisches Wörterbuch. Erarbeitet von einem Autorenkollektiv des Zentralinstituts für Sprachwissenschaft unter der Leitung von Wolfgang Pfeifer. 2. Auflage. Berlin: dtv

Reis, Marga (1983): Gegen die Kompositionstheorie der Affigierung. Zeitschrift fïr Sprachwissenschaft 2,1. 110-131.

Simmler, Franz (1998): Morphologie des Deutschen. Berlin: Weidler Buchverlag

Steche, Theodor (1925): Neue Wege zum reinen Deutsch. Breslau: Ferdinand Hirt

Sugarewa, Tekla (1974): Adjektivderivate zu Eigennamen und ihre Konkurrenz mit Substantivkomposita und syntaktischen Wortverbindungen. Beiträge zur Geschichte der deutschen Sprache und Literatur 94. 199-256

Theissen, S.; Alexis, R.; Kefer, M.; Tewill, G.-T. (1992): Rückläufiges Wörterbuch des Deutschen. Liège: C.I.P.L

Wilmanns, Wilhelm (1930): Deutsche Grammatik: Gotisch, Alt-, Mittel- und Neuhochdeutsch. Zweite Abteilung: Wortbildung. 2. Auflage. Berlin; Lcipzig: Walter dc Gruyter 
Wunderlich, Dieter (1986): Probleme der Wortstruktur. Zeitschrift für Sprachwissenschaft. 5,2: 209-252 Zifonun, Gisela; Hoffmann, Ludger; Strecker, Bruno et al. (1997): Grammatik der deutschen Sprache. Berlin; New York: Walter de Gruyter

Nanna Fuhrhop Universität Potsdam Postfach 601553

14415 Potsdam Germany

fuhrhop@zas.gwz-berlin.de
ZAS Berlin

Jägerstraße 10/11

10117 Berlin

Germany 